J Am Acad Dermatol. 2016 December ; 75(6): 1119-1125.e1. doi:10.1016/j.jaad.2016.07.017.

\title{
Association of atopic dermatitis with smoking: A systematic review and meta-analysis
}

\author{
Robert Kantor, BS ${ }^{\mathrm{a}}$, Ashley Kim, BS ${ }^{\mathrm{a}}$, Jacob Thyssen, MD, $\mathrm{PhD}^{\mathrm{e}}$, and Jonathan I. \\ Silverberg, MD, PhD, MPH ${ }^{a, b, c, d}$ \\ aDepartment of Dermatology, Northwestern University Feinberg School of Medicine, Chicago \\ bDepartment of Preventive Medicine, Northwestern University Feinberg School of Medicine, \\ Chicago \\ 'Department of Medical Social Sciences, Northwestern University Feinberg School of Medicine, \\ Chicago \\ dNorthwestern Medicine Multidisciplinary Eczema Center, Chicago \\ eDepartment of Dermatology, and Allergology, Herlev-Gentofte University Hospital, Hellerup
}

\begin{abstract}
Background-Tobacco exposure might be a modifiable risk factor for atopic dermatitis (AD).

Objective-We examine the association between $\mathrm{AD}$ and exposure to tobacco smoke.

Methods-We performed a systematic review and meta-analysis of observational studies $(n=86)$ in MEDLINE, EMBASE, Scopus, and Cochrane Library (1823-2015). Quality of evidence was assessed using the Newcastle-Ottawa Scale (NOS). A meta-analysis was performed using randomeffects models to estimate pooled odds ratios (OR). Subset analyses were performed for different ages (children, adult), regions, study designs (cross-sectional, longitudinal), study sizes ( $<5000$, 25000), study quality (NOS score <6, $\prec$ ), and amount of smoking (mild, extensive).
\end{abstract}

Results-A diagnosis of AD was associated with higher odds of active smoking (OR 1.87, 95\% confidence interval 1.32-2.63) and exposure to passive smoke (OR 1.18, 95\% confidence interval 1.01-1.38), but not maternal smoking during pregnancy (OR 1.06, 95\% confidence interval 0.80 1.40). The association between active smoking and $\mathrm{AD}$ remained significant in children and adults, all continents studied, and study sizes, but all were cross-sectional designs and had NOS score 6 or greater. Passive smoke was associated with AD in children and adults, cross-sectional studies, South/Central American and African studies, study size less than 5000, and NOS score less than 6.

Limitations-AD severity and distribution were not assessed.

Reprint requests: Jonathan I. Silverberg, MD, PhD, MPH, Department of Dermatology, Northwestern University, 676 N St Clair St, Suite 1600, Chicago, IL 60611. JonathanISilverberg@ gmail.com.

Mr Kantor and Ms Kim contributed equally to this article.

Conflicts of interest: None declared. 
Conclusions-Active and passive exposure to smoke are associated with increased AD prevalence.

\section{Keywords}

active smoking; atopic dermatitis; eczema; meta-analysis; passive smoking; prenatal smoking; prevalence; systematic review

Atopic dermatitis (AD) is a chronic inflammatory skin disorder with multifactorial causes that include genetic predisposition, immune dysregulation, and external environmental factors. ${ }^{1,2}$ The prevalence of AD has increased over the past 50 years in much of the world and continues to increase in many countries. ${ }^{3}$ The US prevalence of AD has recently been estimated to be $12.2 \%$ in children ${ }^{4}$ and $7 \%$ to $10 \%$ in adults. ${ }^{5,6}$ It is imperative to identify modifiable risk factors to reduce the prevalence of disease.

Tobacco smoke has a number of harmful effects on the immune system, ${ }^{7}$ eg, on humoral and cellular immunity. The putative direct effect of tobacco smoke on the skin is unclear, ${ }^{8}$ but smoke might directly impair skin-barrier function via the effects of reactive oxygen species on keratinocytes. ${ }^{9,10}$ Numerous studies explored the relationship between tobacco smoke and various atopic diseases including asthma, allergic rhinitis, allergic conjunctivitis, and AD. In particular, epidemiologic studies demonstrated a strong link between environmental tobacco smoke and asthma in the pediatric population, with an increased incidence of asthma and wheeze by at least $20 \% .{ }^{11}$ Furthermore, smoke-free legislation has been associated with lower rates of hospital visits for asthma. ${ }^{12}$ In contrast, a prospective population-based study found a decreased risk of IgE-mediated allergic sensitization in sustained smokers compared with never smokers. ${ }^{13}$

Recent studies demonstrated that US adults with AD had increased cardiovascular disease including hypertension, adult-onset diabetes and hypercholesterolemia, coronary artery disease, heart attack, stroke, and peripheral vascular disease. ${ }^{14,15}$ In these studies, adult AD was associated with increased cigarette smoking and other poor health behaviors. Similarly, a Danish study found that adults with $\mathrm{AD}$ had higher risk for cardiovascular disease, ${ }^{16}$ which appeared to be caused by poor health behaviors. Thus, higher rates of smoking and other tobacco exposure in patients with $\mathrm{AD}$ is of utmost importance to cardiovascular risk and other health outcomes.

We performed a systematic review and meta-analysis to determine whether smoking is associated with a diagnosis of AD. In particular, we examined the relationship of AD with active smoking, exposure to passive smoke, and maternal smoking during pregnancy.

\section{METHODS}

\section{Literature search}

We searched the following databases through March 1, 2015: PubMed (1946-present), EMBASE (1947-present), Scopus (1823-present), and the Cochrane Library (1992-present). The search strategy was based on a previous Cochrane review ${ }^{17}$ with inclusion of "smoking," "cigarette," "nicotine," and "tobacco" as additional search terms (Supplemental 
Table I; available at http://www.jaad.org). Studies published online and in print and in press from all years were considered. All search results with titles and abstracts written in any language were eligible for inclusion. Studies were excluded based on the title, abstract, or both if there was no clear indication they were investigating exposure to tobacco smoke in patients with $\mathrm{AD}$ or if they were case series, ie, there was no control group without $\mathrm{AD}$.

All studies with primary epidemiologic data reporting the relationship between $\mathrm{AD}$ and active smoking, passive smoke exposure in the home, maternal smoking during pregnancy, or a combination of these were included. If data were duplicated in more than 1 study, the most recent and complete study was included in the meta-analysis.

\section{Data extraction}

Two reviewers (A. K., R. K.) independently performed data extraction from these studies and any differences were resolved by discussion. From these 86 studies, data items collected were: study design; how AD was diagnosed; how smoking was assessed; country of study; total number of patients or individuals in study; number of individuals with $\mathrm{AD}$, exposure to active smoking, passive smoking in the home, and/or maternal smoking during pregnancy; severity of AD divided into mild, moderate, or severe; mean age of patients; and percent of males in the study. Fifteen studies presented total group sizes for the exposed and nonexposed groups to smoking and the odds ratios (OR) of AD for each type of smoke exposure. The prevalence of $\mathrm{AD}$ within each type of smoking was reconstructed as previously described. ${ }^{18}$

AD was defined differently throughout studies, where only 12 used physician diagnosis with the Hanifin and Rajka ${ }^{19}$ or other criteria; the other 74 studies used combinations of various questions from the International Study of Asthma and Allergies in Childhood, ${ }^{20}$ or other definitions. When data related to multiple approaches for the assessment of AD were presented, we selected the preferred definition of $\mathrm{AD}$ in the following order: diagnosis by criteria of Hanifin and Rajka, ${ }^{19}$ other health care diagnosis of AD, self-report of ever being diagnosed by a physician with eczema, presence of an itchy rash in the typical (flexural) distribution, ever history of eczema, and other definitions. To address the varied definitions of the exposure and outcomes, we performed sensitivity analyses by location of study and by children versus adults. Smoking was defined predominantly via self- and caregiver-report, with virtually no studies directly using proxy measures of exposure in people or air.

The Newcastle-Ottawa Scale (NOS) for assessing the quality of nonrandomized studies in meta-analysis was used. ${ }^{21}$ The scoring system summarized 8 aspects of each study: case definition adequacy, representativeness of the cases, selection of controls, definition of controls, comparability of cases and controls on the basis of the design or analysis, ascertainment of exposure, same method of ascertainment for cases and controls, and comparison of nonresponse rate between cases and controls. High-quality studies were defined as a score 6 or more of 9 total points. Sensitivity analyses were performed for studies with NOS score less than 6 or 6 or higher. 


\section{Statistical analysis}

Given the relative dearth of well-constructed studies regarding this topic, we decided a priori to include all studies in the meta-analysis regardless of study quality. Our statistical analyses were performed using software (SAS, Version 9.4, SAS Institute Inc, Cary, NC).

Comparable outcomes of all studies of AD and active smoking, passive exposure to smoke in the home, and maternal smoking during pregnancy were combined, and measures of consistency were obtained. Significant heterogeneity of results was detected across studies as judged by a Cochrane Q statistic $P$ value less than $.05, P^{2}$ statistic greater than $50 \%$, or both. Therefore, random-effect models were performed that accounted for variability between studies. A 2 -sided $P$ value of .05 was taken as significant. Begg rank correlation and funnel plot regression were used to assess for potential publication bias.

\section{RESULTS}

\section{Literature search}

The literature search yielded 5817 nonduplicate manuscripts. After review of the titles and abstracts, 5283 were excluded; an additional 448 manuscripts were excluded after full-text review. In total, 86 studies were included in the review, of which 20 had valid data to assess for the effects of active smoking, 66 for passive exposure to tobacco smoke, and 23 for maternal smoking during pregnancy. The Preferred Reporting Items for Systematic Reviews and Meta-Analyses (PRISMA) flow diagram is presented in Fig 1.

\section{Study characteristics}

The studies were cross-sectional or longitudinal with respect to prevalence of $\mathrm{AD}$, used either retrospective or prospective data collection, included both males and/or females, and encompassed participants of all ages (a listing of the studies included in the analysis is available on request from the authors). Published years of studies ranged from 1989 to 2015. The 86 studies included 680,176 patients from 39 countries, including 598,296 children and 67,501 adults with an additional 14,379 nonspecified children or adults, of which 65,173 had $\mathrm{AD}$ (9.6\% of the entire cohort). Quality assessment using the NOS scale revealed that 66 of the 86 included studies had scores of 6 or greater (raw data available on request from the authors).

Overall, 17,969 (12.9\%; range 1.2\%-50.0\%) of patients who were active smokers, 33,200 (15.3\%; range $0.9 \%-56.8 \%$ ) of patients who were passively exposed to tobacco smoke in the home, and 14,004 (15.4\%; range $2.3 \%-34.4 \%)$ of children who were born to mothers who smoked during pregnancy, respectively, had a previous and/or current diagnosis of AD. Significant heterogeneity was identified between studies of active smoking, passive smoke exposure, and maternal smoking during pregnancy, as judged by 2 different measures of heterogeneity (Cochrane Q $P$ value $<.0001$ and $P^{2} \geq 74.7 \%$ for all 3 outcomes).

\section{Association between active smoking and $A D$}

In random-effects models, $\mathrm{AD}$ was associated with active smoking (OR 1.87, 95\% confidence interval [CI] 1.32-2.63) (Table I). The association between active smoking and AD prevalence remained significant in studies of both children (OR 2.19, 95\% CI 1.34- 
3.57) and adults (OR 1.30, 95\% CI 1.06-1.59), across all regions (North America: OR 1.66, 95\% CI 1.42-1.93; Asia: OR 1.74, 95\% CI 1.05-2.88; Africa: OR 8.41, 95\% CI 3.66-19.32; Europe: OR 1.91, 95\% CI 1.16-3.13), irrespective of study size ( $<$ < 5000: OR 2.08, 95\% CI $1.31-3.32 ; \mathrm{n} \geq 5000$ : OR $1.68,95 \%$ CI 1.09-2.53). All studies of active smoking were crosssectional and had NOS score of 6 or greater.

Only 2 studies stratified AD prevalence by the amount of active smoking. ${ }^{22,23} \mathrm{AD}$ was significantly associated with both mild (OR 2.68, 95\% CI 1.78-4.04) and extensive (OR $2.70,95 \%$ CI 1.11-6.60) amounts of active smoking with similar effect sizes. No studies examined the relationship between AD severity and active smoking.

\section{Association between passive exposure to smoke in the home and AD}

$\mathrm{AD}$ was also associated with passive exposure to smoking in the home (OR 1.18, 95\% CI 1.01-1.38). Passive exposure to smoke appeared to have a stronger effect in adults (OR 3.62, 95\% CI 1.71-7.69) than children (OR 1.15, 95\% CI 1.01-1.30). The association between passive smoke exposure and AD prevalence remained significant in studies from South/ Central America (OR 1.95, 95\% CI 1.55-2.46) and Africa (OR 2.23, 95\% CI 1.19-4.19), but only marginal significance in Asia (OR $1.22,95 \%$ CI $0.98-1.51$ ) and no significance in North America (OR 1.29, 95\% CI 0.94-1.76) or Europe (OR 0.96, 95\% CI 0.75-1.22). Moreover, the association between $\mathrm{AD}$ and passive smoking was only significant in studies with sample size less than 5000 (OR 1.30, 95\% CI 1.07-1.59), not 5000 or greater (OR 1.02, 95\% CI 0.81-1.29).

Eleven studies examined stratified AD prevalence by the levels of exposure to passive smoking. ${ }^{23-33}$ There were no significant associations between $\mathrm{AD}$ and exposure to mild (OR $1.21,95 \%$ CI $0.81-1.80$ ) or extensive (OR $1.08,95 \%$ CI $0.74-1.59$ ) amounts of passive smoking.

One study examined the relationship between AD severity and passive smoking and found significantly higher rates of heavier smoking in mild and severe AD compared with no AD $\left(42.1 \%, 40.0 \%\right.$, and $25.3 \%$, respectively). ${ }^{34}$

\section{Association between maternal smoking during pregnancy and $A D$}

Finally, AD was not significantly associated with maternal smoking during pregnancy overall (OR 1.06, 95\% CI 0.80-1.40); all studies were conducted in children and not adults. However, there were conflicting results between sensitivity analyses. There was a positive association between $\mathrm{AD}$ and maternal smoking during pregnancy in studies from Asia (OR $1.59,95 \%$ CI 1.28-2.03) and a NOS score less than 6 (OR 1.96, 95\% CI 1.50-2.64), but an inverse association seen in studies with sample size 5000 or greater (OR $0.77,95 \%$ CI 0.58 $0.93)$.

Only 5 studies stratified AD prevalence by the amount of maternal smoking during pregnancy. ${ }^{24,35-38}$ There were no significant associations between AD prevalence and the amount of maternal smoking during pregnancy. No studies examined the relationship between $\mathrm{AD}$ severity and maternal smoking during pregnancy. 


\section{Publication bias}

Publication bias was not detected as judged by nonsignificant Begg rank correlation or funnel plot regression for any of the above-mentioned outcomes $(P \geq .21)$.

\section{DISCUSSION}

The results of this meta-analysis of observational studies suggest that active smoking and passive exposure to cigarette smoke in the home are associated with higher prevalence of $\mathrm{AD}$ in both children and adults. In contrast, maternal smoking during pregnancy was not associated with $\mathrm{AD}$ prevalence overall and showed inconsistent associations in sensitivity analyses. Although AD was associated with both mild and extensive amounts of active smoking, there was no dose-response relationship observed. All studies of active smoking were cross-sectional, which precludes determination of whether smoking causes $\mathrm{AD}$ or vice versa. It could be that even minimal amounts of active smoking are harmful in patients with $\mathrm{AD}$. Alternatively, the negative emotional effects of $\mathrm{AD}$ may result in increased rates of smoking, irrespective of the amount of smoke exposure. The lack of association between $\mathrm{AD}$ and passive smoking or maternal smoking during pregnancy in available cohort studies suggests that exposure to smoke may not trigger $\mathrm{AD}$ per se. Rather, there may be confounding factors, such as race/ethnicity, education, and socioeconomic status, that account for such associations. Of note, there were regional differences for the association between passive exposure to smoke and maternal smoking during pregnancy. Some of these differences may be related to public health policy or cultural differences, eg, prohibition against smoking indoors, versus climate differences, eg, increased humidity. Regardless, the results of this meta-analysis suggest that pediatric and adult patients with AD have higher rates of active and passive smoke exposure, which might increase their risk for malignancy and cardiovascular disease. ${ }^{14-16}$ The mechanism behind the association of smoking and AD remains unknown. It is likely that smoking has multiple effects on immune responses and skin-barrier function that may contribute to $\mathrm{AD}$.

Previous studies have shown that adverse childhood experiences negatively impact health behaviors later in life and may lead to higher rates of smoking. ${ }^{39}$ It may be that AD has similar detrimental effects. Indeed, 1 study found that adults with AD had a significantly earlier age of smoking onset compared with those without AD. ${ }^{15}$ Interestingly, passive exposure to smoke was associated with a stronger effect in adults than children, perhaps owing to delayed disease manifestation with a cumulative dose-response effect of smoke exposure. ${ }^{40}$ Further investigation of the impact of passive smoke exposure on age of $\mathrm{AD}$ onset would be useful to explore this observation. Because smoking is associated with higher prevalence of $\mathrm{AD}$, it is quite possible that smoking is also associated with more severe AD. However, virtually no studies assessed the effects of smoking on AD severity. Thus, future studies are warranted to determine whether smoking is associated with more severe $\mathrm{AD}$ and whether smoking cessation improves disease severity.

There are multiple strengths to this meta-analysis, including the use of random-effect models to address heterogeneity among studies and multiple sensitivity analyses. Most of the included studies were high quality and conducted in multiple countries. However, this study has some limitations. The pooled studies had different inclusion/exclusion criteria, sample 
sizes, sampling approaches, and regions. Moreover, the included studies were predominantly cross-sectional, which limits the ability to determine a temporal relationship between smoking and AD. No cohort studies examined whether active smoking triggered AD. Most studies of maternal smoking during pregnancy did not provide information about trimester(s) of smoke exposure. Moreover, virtually no studies examined the effects of smoking on AD severity. Most studies relied on self-report of smoking status rather than objective measurements of indoor smoke or metabolites of smoke exposure in participants. The effects of smoking on $\mathrm{AD}$ may be modified by other factors, such as regional differences of smoking practices, climate, or both. To address these potential regional differences, we stratified models by region and there were significant differences. However, there were insufficient data to allow for further exploration of such regional differences. Finally, the association with smoke could be confounded by stress and neuropsychiatric disorders, which are also associated with AD. ${ }^{41-43}$

In conclusion, active smoking and passive smoke exposure are associated with increased prevalence of $\mathrm{AD}$, in children and adults. Future studies are needed to address knowledge gaps in the association of smoking and $\mathrm{AD}$, including studies using objective measures of AD severity such as the Eczema Area and Severity Index, objective measures of smoke exposure, more longitudinal studies to determine the temporal relationship between smoking and $\mathrm{AD}$, and studies to explore the mechanisms of association. Finally, future studies are needed to determine the contribution of smoking toward cardiovascular risk in patients with $\mathrm{AD}$.

\section{Supplementary Material}

Refer to Web version on PubMed Central for supplementary material.

\section{Acknowledgments}

This publication was made possible with support from the Agency for Healthcare Research and Quality, grant number K12HS023011, and the Dermatology Foundation. No honorarium, grant, or other form of individual payment was given to anyone to produce the manuscript.

\section{Abbreviations used}

$\begin{array}{ll}\text { AD } & \text { atopic dermatitis } \\ \text { CI } & \text { confidence interval } \\ \text { NOS } & \text { Newcastle-Ottawa Scale } \\ \text { OR } & \text { odds ratio }\end{array}$

\section{References}

1. Thomsen SF. Atopic dermatitis: natural history, diagnosis, and treatment. ISRN Allergy. 2014; 2014:354250. [PubMed: 25006501]

2. Zhang A, Silverberg JI. Association of atopic dermatitis with being overweight and obese: a systematic review and metaanalysis. J Am Acad Dermatol. 2015; 72:606-616. e4. [PubMed: 25773409] 
3. Deckers IA, McLean S, Linssen S, Mommers M, van Schayck CP, Sheikh A. Investigating international time trends in the incidence and prevalence of atopic eczema 1990-2010: a systematic review of epidemiological studies. PLoS One. 2012; 7:e39803. [PubMed: 22808063]

4. Silverberg JI, Hanifin J, Simpson EL. Climatic factors are associated with childhood eczema prevalence in the United States. J Invest Dermatol. 2013; 133:1752-1759. [PubMed: 23334343]

5. Silverberg JI, Garg NK, Paller AS, Fishbein AB, Zee PC. Sleep disturbances in adults with eczema are associated with impaired overall health: a US population-based study. J Invest Dermatol. 2015; 135:56-66. [PubMed: 25078665]

6. Silverberg JI, Hanifin JM. Adult eczema prevalence and associations with asthma and other health and demographic factors: a US population-based study. J Allergy Clin Immunol. 2013; 132:1132_ 1138. [PubMed: 24094544]

7. Sopori M. Effects of cigarette smoke on the immune system. Nat Rev Immunol. 2002; 2:372-377. [PubMed: 12033743]

8. Frohlich M, Sund M, Lowel H, Imhof A, Hoffmeister A, Koenig W. Independent association of various smoking characteristics with markers of systemic inflammation in men. Results from a representative sample of the general population (MONICA Augsburg Survey 1994/95). Eur Heart J. 2003; 24:1365-1372. [PubMed: 12871694]

9. Sticozzi C, Belmonte G, Pecorelli A, et al. Cigarette smoke affects keratinocytes SRB1 expression and localization via $\mathrm{H} 2 \mathrm{O} 2$ production and HNE protein adducts formation. PLoS One. 2012; 7:e33592. [PubMed: 22442701]

10. Egawa M, Kohno Y, Kumano Y. Oxidative effects of cigarette smoke on the human skin. Int J Cosmet Sci. 1999; 21:83-98. [PubMed: 18505533]

11. Burke H, Leonardi-Bee J, Hashim A, et al. Prenatal and passive smoke exposure and incidence of asthma and wheeze: systematic review and meta-analysis. Pediatrics. 2012; 129:735-744. [PubMed: 22430451]

12. Been JV, Nurmatov UB, Cox B, Nawrot TS, van Schayck CP, Sheikh A. Effect of smoke-free legislation on perinatal and child health: a systematic review and meta-analysis. Lancet. 2014; 383:1549-1560. [PubMed: 24680633]

13. Linneberg A, Nielsen NH, Madsen F, Frolund L, Dirksen A, Jorgensen T. Smoking and the development of allergic sensitization to aeroallergens in adults: a prospective population-based study. The Copenhagen Allergy Study. Allergy. 2001; 56:328-332. [PubMed: 11284801]

14. Silverberg JI. Association between adult atopic dermatitis, cardiovascular disease, and increased heart attacks in three population-based studies. Allergy. 2015; 70:1300-1308. [PubMed: 26148129]

15. Silverberg JI, Greenland P. Eczema and cardiovascular risk factors in 2 US adult population studies. J Allergy Clin Immunol. 2015; 135:721-728. e6. [PubMed: 25579484]

16. Andersen YMF, Egeberg A, Gislason GH, Hansen PR, Skov L, Thyssen JP. Risk of myocardial infarction, ischemic stroke, and cardiovascular death in patients with atopic dermatitis. J Allergy Clin Immunol. 2016; 138:310-312. e3. [PubMed: 26971689]

17. Nankervis H, Pynn EV, Boyle RJ, et al. House dust mite reduction and avoidance measures for treating eczema. Cochrane Database of Systematic Reviews. 2015; (1):CD008426. [PubMed: 25598014]

18. Di Pietrantonj C. Four-fold table cell frequencies imputation in meta-analysis. Stat Med. 2006; 25:2299-2322. [PubMed: 16025540]

19. Hanifin J, Rajka G. Diagnostic features of atopic dermatitis. Acta Derm Venerol (Stockh). 1980; 92:44-47.

20. The International Study of Asthma and Allergies in Childhood (ISAAC) Steering Committee. Worldwide variation in prevalence of symptoms of asthma, allergic rhinoconjunctivitis, and atopic eczema: ISAAC. Lancet. 1998; 351:1225-1232. [PubMed: 9643741]

21. Stang A. Critical evaluation of the Newcastle-Ottawa scale for the assessment of the quality of nonrandomized studies in meta-analyses. European Journal of Epidemiology. 2010; 25:603-605. [PubMed: 20652370] 
22. Graif Y, German L, Ifrah A, Livne I, Shohat T. Dose-response association between smoking and atopic eczema: results from a large cross-sectional study in adolescents. Dermatology. 2013; 226:195-199. [PubMed: 23711459]

23. Tanaka K, Miyake Y, Arakawa M. Smoking and prevalence of allergic disorders in Japanese pregnant women: baseline data from the Kyushu Okinawa Maternal and Child Health Study. Environ Health. 2012; 11:15. [PubMed: 22413964]

24. Apfelbacher CJ, Diepgen TL, Schmitt J. Determinants of eczema: population-based cross-sectional study in Germany. Allergy. 2011; 66:206-213. [PubMed: 20804468]

25. Ergin S, Ozsahin A, Erdogan BS, Aktan S, Zencir M. Epidemiology of atopic dermatitis in primary schoolchildren in Turkey. Pediatr Dermatol. 2008; 25:399-401. [PubMed: 18577060]

26. Foliaki S, Annesi-Maesano I, Tuuau-Potoi N, et al. Risk factors for symptoms of childhood asthma, allergic rhinoconjunctivitis and eczema in the Pacific: an ISAAC phase III study. Int J Tuberc Lung Dis. 2008; 12:799-806. [PubMed: 18544207]

27. Heudorf U, Schumann M, Angerer J, Exner M. Dermal and bronchial symptoms in children: are they caused by PAH containing parquet glue or by passive smoking? Int Arch Occup Environ Health. 2005; 78:655-662. [PubMed: 16001207]

28. Hjern A, Hedberg A, Haglund B, Rosen M. Does tobacco smoke prevent atopic disorders? A study of two generations of Swedish residents. Clin Exp Allergy. 2001; 31:908-914. [PubMed: 11422156]

29. Lee YL, Li CW, Sung FC, Guo YL. Increasing prevalence of atopic eczema in Taiwanese adolescents from 1995 to 2001. Clin Exp Allergy. 2007; 37:543-551. [PubMed: 17430351]

30. Morales Suarez-Varela M, Garcia-Marcos L, Kogan MD, et al. Parents' smoking habit and prevalence of atopic eczema in 6-7 and 13-14 year-old schoolchildren in Spain. ISAAC phase III. Allergol Immunopathol. 2008; 36:336-342.

31. Song N, Shamssain M, Zhang J, et al. Prevalence, severity and risk factors of asthma, rhinitis and eczema in a large group of Chinese schoolchildren. J Asthma. 2014; 51:232-242. [PubMed: 24303994]

32. Suarez-Medina R, Venero-Fernandez SJ, de la Mora-Faife E, et al. Risk factors for eczema in infants born in Cuba: a population-based cross-sectional study. BMC Dermatol. 2014; 14:6. [PubMed: 24666750]

33. Tanaka K, Miyake Y. Association between prenatal and postnatal tobacco smoke exposure and allergies in young children. J Asthma. 2011; 48:458-463. [PubMed: 21548833]

34. Sugiyama T, Sugiyama K, Toda M, Yukawa T, Makino S, Fukuda T. Risk factors for asthma allergic diseases among 13-14-year-old schoolchildren in Japan. Allergol Int. 2002; 51:139-150.

35. Bohme M, Kull I, Bergstrom A, et al. Parental smoking increases the risk for eczema with sensitization in 4-year-old children. J Allergy Clin Immunol. 2010; 125:941-943. [PubMed: 20227755]

36. Linneberg A, Simonsen JB, Petersen J, Stensballe LG, Benn CS. Differential effects of risk factors on infant wheeze and atopic dermatitis emphasize a different etiology. J Allergy Clin Immunol. 2006; 117:184-189. [PubMed: 16387604]

37. Magnusson LL, Olesen AB, Wennborg H, Olsen J. Wheezing, asthma, hayfever, and atopic eczema in childhood following exposure to tobacco smoke in fetal life. Clin Exp Allergy. 2005; 35:15501556. [PubMed: 16393320]

38. Yi O, Kwon HJ, Kim H, et al. Effect of environmental tobacco smoke on atopic dermatitis among children in Korea. Environ Res. 2012; 113:40-45. [PubMed: 22264877]

39. Campbell JA, Walker RJ, Egede LE. Associations between adverse childhood experiences, highrisk behaviors, and morbidity in adulthood. Am J Prev Med. 2016; 50:344-352. [PubMed: 26474668]

40. Lee $\mathrm{CH}$, Chuang HY, Hong $\mathrm{CH}$, et al. Lifetime exposure to cigarette smoking and the development of adult-onset atopic dermatitis. Br J Dermatol. 2011; 164:483-489. [PubMed: 21054333]

41. Michal M, Wiltink J, Reiner I, et al. Association of mental distress with smoking status in the community: results from the Gutenberg Health Study. J Affect Disord. 2013; 146:355-360. [PubMed: 23063238] 
42. Yu SH, Silverberg JI. Association between atopic dermatitis and depression in US adults. J Invest Dermatol. 2015; 135:3183-3186. [PubMed: 26316069]

43. Garg N, Silverberg JI. Association between childhood allergic disease, psychological comorbidity, and injury requiring medical attention. Ann Allergy Asthma Immunol. 2014; 112:525-532.

[PubMed: 24713577] 


\begin{tabular}{|c|c|}
\hline $\begin{array}{c}\text { MEDLINE (1946-Mar 2015): } \\
964 \text { citations }\end{array}$ & $\begin{array}{c}\text { GREAT (2000-Mar 2015): } \\
5 \text { citations }\end{array}$ \\
\hline \hline $\begin{array}{c}\text { EMBASE (1947-Mar 2015): } \\
\text { 1810 citations }\end{array}$ & $\begin{array}{c}\text { SCOPUS (1823-Mar 2015): } \\
\text { 8558 citations }\end{array}$ \\
\hline Cochrane Library \\
(1992-Mar 2015): \\
141 citations
\end{tabular}

5817 non-duplicate citations screened

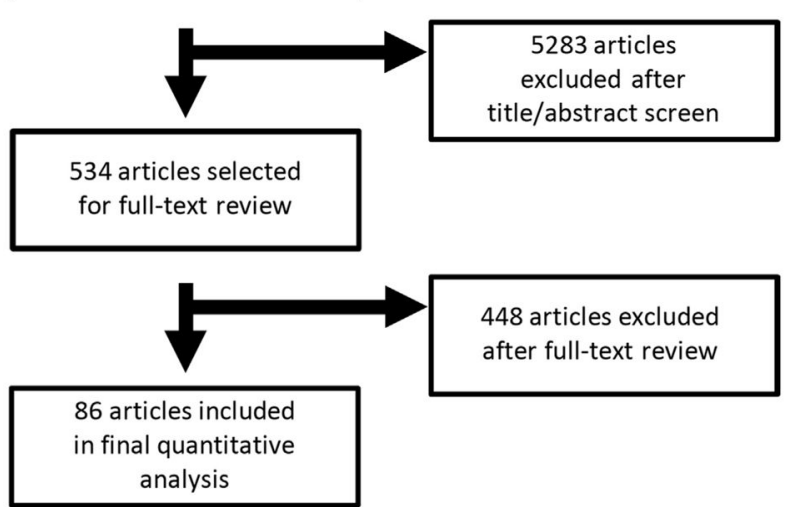

Fig 1.

PRISMA flow diagram of literature search and study selection for meta-analysis of atopic dermatitis and smoking. 


\section{Table I}

Random-effect models and sensitivity analyses for the association between smoking and atopic dermatitis

\begin{tabular}{|c|c|c|c|}
\hline \multirow[b]{2}{*}{ Study subsets } & \multicolumn{3}{|c|}{ OR $(95 \% \mathrm{CI})$} \\
\hline & Active smoking, $\mathrm{n}=\mathbf{2 0}$ & Passive smoke exposure, $n=66$ & Maternal smoking during pregnancy, $\mathrm{n}=$ \\
\hline Overall & $1.87(1.32-2.63)$ & $1.18(1.01-1.38)$ & $1.06(0.80-1.40)$ \\
\hline \multicolumn{4}{|l|}{ Age } \\
\hline Children $<18$ y & $2.19(1.34-3.57)$ & $1.15(1.01-1.30)$ & $1.02(0.81-1.27)$ \\
\hline Adults $\geq 18 \mathrm{y}$ & $1.30(1.06-1.59)$ & $3.62(1.71-7.69)$ & N/A \\
\hline \multicolumn{4}{|l|}{ Study design } \\
\hline Case-control/cross-sectional & $1.87(1.32-2.63)$ & $1.19(1.01-1.41)$ & $1.14(0.79-1.65)$ \\
\hline Cohort/longitudinal & N/A & $1.13(0.79-1.61)$ & $0.93(0.65-1.34)$ \\
\hline \multicolumn{4}{|l|}{ Region } \\
\hline North America & $1.66(1.42-1.93)$ & $1.28(0.94-1.76)$ & $0.49(0.15-1.65)$ \\
\hline South/Central America & N/A & $1.95(1.55-2.46)$ & N/A \\
\hline Asia & $1.74(1.05-2.88)$ & $1.22(0.98-1.51)$ & $1.59(1.28-2.03)$ \\
\hline Africa & $8.41(3.66-19.32)$ & $2.23(1.19-4.19)$ & N/A \\
\hline Europe & $1.91(1.16-3.13)$ & $0.96(0.75-1.22)$ & $0.94(0.68-1.10)$ \\
\hline \multicolumn{4}{|l|}{ Study size } \\
\hline$<5000$ & $2.08(1.31-3.32)$ & $1.30(1.07-1.59)$ & $1.37(0.94-1.95)$ \\
\hline 25000 & $1.68(1.09-2.53)$ & $1.02(0.81-1.29)$ & $0.77(0.58-0.93)$ \\
\hline \multicolumn{4}{|l|}{ NOS score } \\
\hline$<6$ & N/A & $1.18(1.11-1.26)$ & $1.96(1.50-2.64)$ \\
\hline$\checkmark 6$ & $1.87(1.32-2.63)$ & $1.11(0.91-1.36)$ & $0.88(0.67-1.16)$ \\
\hline Amount of smoking & $(\mathrm{n}=2)$ & $(\mathbf{n}=\mathbf{1 1})$ & $(\mathbf{n}=5)$ \\
\hline Mild & $2.68(1.78-4.04)$ & $1.21(0.81-1.80)$ & $1.09(0.68-1.72)$ \\
\hline Extensive & $2.70(1.11-6.60)$ & $1.08(0.74-1.59)$ & $0.74(0.36-1.52)$ \\
\hline
\end{tabular}

Bold indicates statistically significant associations $(P<.05)$. Random-effect models were performed with history of atopic dermatitis as the dependent variable and active smoking, passive smoke exposure, or neonatal smoke exposure as the independent variables. Pooled OR and $95 \% \mathrm{CI}$ were calculated. Sensitivity analyses were performed for children vs adults, North America vs South America vs Asia vs Africa vs Europe, study size $<5000$ vs 25000 , Newcastle-Ottawa Scale of $<6$ vs $\prec 6$, and mild vs extensive smoking.

$C I$, Confidence interval; N/A, not available; NOS, Newcastle-Ottawa Scale; $O R$, odds ratio. 